\title{
Katholiken am wenigsten selbstmordgefährdet
} \section{Vorsicht bei der Berufswahl!}

JUGENDLICHE NEURODERMITIKER

- In einer prospektive Studie mit fast 4000 Kindern gingen Forscher der Frage nach, wie sich eine atopische Dermatitis in der Pubertät entwickelt und welche Risikofaktoren dabei eine besondere Rolle spielen. Etwa $7 \%$ der Teilnehmer entwickelten erst in der Jugend eine atopische Dermatitis. Mädchen waren davon häufiger betroffen als Jungen.

Ein wichtiger Risikofaktor war bei den Jugendlichen - neben engen Verwandten mit einer Neurodermitis - der berufliche

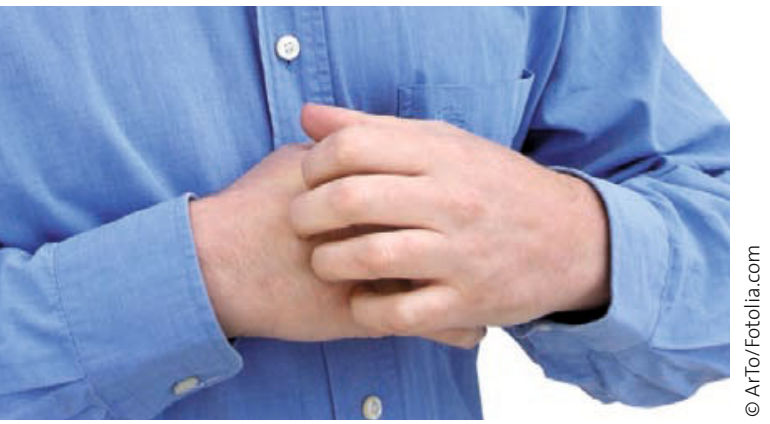

Bäckermehl und Desinfektionsmittel fördern die Neurodermitis.

Kontakt zu reizauslösenden Substanzen, z.B. Bäckermehl oder Desinfektionsmittel. Es zeigte sich auch, dass Risikofaktoren, die im Säuglings- und Kindesalter eine wichtige Rolle spielten (u. a. dass ein Kind nicht gestillt wurde, keine Geschwister hat und keinen Kindergarten besuchte), in der Pubertät an Bedeutung verlieren. Ärzte sollten ihre jugendlichen Neurodermitiker deshalb von Hochrisikoberufen wie Bäcker, Reinigungskraft oder Krankenpfleger abraten, empfehlen die Studienautoren.

KE =

- Pressemitteilung der LMU München; J Allergy Clin Immunol. 2010;126(3):590-595.e3

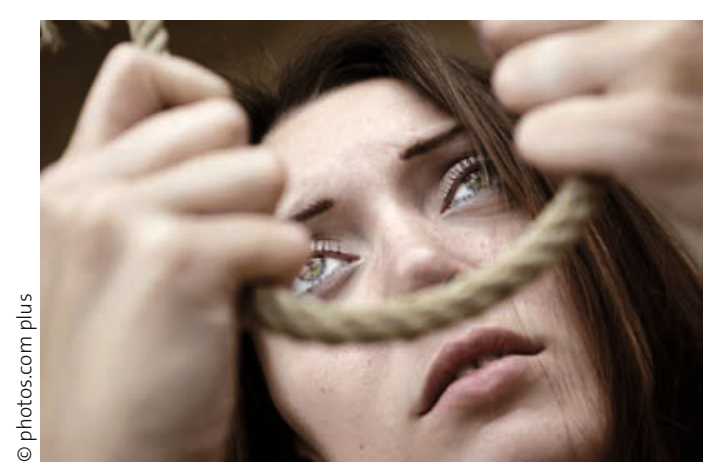

- Je ferner ein Mensch der Religion steht, desto suizidgefährdeter scheint er zu sein. Zu diesem Ergebnis kommt zumindest eine Kohortenstudie der Universitätsinstitute für Sozial- und Präventivmedizin (ISPM) Bern und Zürich mit drei Millionen Schweizern zwischen 35 und 94 Jahren, basierend auf der Volkszählung 2000. Auf 100000 EinFehlt ihr der Glaube? Menschen, die keiner Glaubensrichtung angehörten. Bei den Protestanten lag die entsprechende Zahl bei 29, und unter den Mitgliedern der römisch-katholischen Kirche nahmen sich 20 Menschen das Leben. Diese Ergebnisse zeigen nach Ansicht der Studienautoren, dass die Zugehörigkeit zu einer Glaubensgemeinschaft eine „wichtige soziale Kraft“ darstellt.

Darüber hinaus konnte in der Studie beobachtet werden, dass sich sowohl der suizidpräventive Effekt der Religion bei den Katholiken als auch die höhere Suizidrate bei den Konfessionslosen mit zunehmendem Alter weiter verstärken. Bei älteren nicht religiösen Menschen war vor allem eine Steigerung bei den assistierten Suiziden zu verzeichnen, die in der Schweiz von einer Sterbehilfeorganisation unterstützt werden.

\section{EMPFEHLUNG VON OTC-PRÄPARATEN Die meisten Ärzte greifen zum Grünen Rezept}

— Wenn GKV-Patienten eine Empfehlung für ein rezeptfreies Arzneimittel wünschen, stellt die Mehrheit der Ärzte ein Grünes Rezept aus. Das ist das Ergebnis einer Befragung der „Ärzte Zeitung“, an der 380 Ärzte teilgenommen haben. Damit wurden die Resultate einer gleichlautenden Umfrage im Januar 2009 bestätigt.

86,2\% der Antwortenden wählen das Grüne Rezept, bei der ersten Umfrage waren es $82,3 \%$. Der Anteil derjenigen, die auf ein blaues Privatrezept setzen, ist mit 14,1\% (2009: 13,6\%) fast konstant blieben. Deutlich abgenommen hat mit 6,9\% die Gruppe der Ärzte, die auf eine mündliche Empfehlung vertrauen (13,9\% in 2009). Gefragt nach den Gründen für das Aus- stellen eines Grünen Rezepts, wird von den Ärzten die Merkhilfe für den Patienten als der wichtigste Aspekt gesehen (71,9\%). 67,1\% der Ärzte geben an, dass dadurch die Arzt-Patienten-Bindung gestärkt wird

Mit dem Grünen Rezept „bekräftige ich meine Therapiehoheit", sagt der Allgemeinarzt Werner Gerhold aus NortheimLangenholtensen. Die Internistin Jutta Eva Nostitz aus Berlin weist darauf hin, das Rezept könne in der Steuererklärung als Beleg für besondere Aufwendungen angeführt werden. Die Allgemeinärztin Dr. Dagmar Aßmann aus Bielefeld stelt fest, dass die Initiative zur Empfehlung meistens von ihr, nicht von ihren Patienten ausgeht. 